\title{
Aspekte moderner Weiterbildung
}

$\mathrm{D}$ er gebetsmühlenartige Gebrauch des im modernen Bildungsbetrieb häufig benutzten Begriff-Dreierpacks Aus-, Fort- und Weiterbildung darf nicht davon ablenken, dass sich dahinter nicht selten ein inhaltlich und konzeptionell schwach oder ungenau formulierter Handlungskatalog verbirgt. Klar ist der Begriff Ausbildung, der die berufliche und wissenschaftliche Vermittlung einer bestimmten Disziplin bezeichnet. Die beiden Begriffe Fort- und Weiterbildung werden dagegen oft synonym gebraucht, obgleich sie eine doch deutlich verschiedene Bedeutung haben. So dient die Fortbildung der Vermittlung von Wissensinhalten, die nicht an eine bestimmte berufliche Zielsetzung gekoppelt ist und schon gar nicht die breite Vermittlung spezifischer Wissensinhalte einer spezifischen Disziplin bedeutet. Im Gegensatz dazu soll die Weiterbildung zur beruflichen disziplinorientierten fachlichen Spezialisierung beitragen, die vor allem im medizinischen Bereich durch die Bundesärztekammer fachbezogen und inhaltlich präzisiert in der Weiterbildungsordnung niedergelegt ist.

Die Fortbildung im Allgemeinen aber auch die wissenschaftliche Weiterbildung im Besonderen haben in den letzten Jahrzehnten gewaltige Veränderungen erfahren. So wächst nicht nur der Umfang an Erkenntnissen und Daten exponentiell, wobei dem Wissen häufig unzulässigerweise eine kurze Halbwertszeit angeheftet wird. Vor allem die Erkenntnisse aus den Forschungsund Arbeitsbereichen, die nicht mehr einer spezifischen, klassischen Disziplin zugeordnet werden können - da sie mit neuen Arbeitstechniken und neuen Forschungsverfahren wissensrelevante Fakten schaffen und typischerweise fachübergreifend mehrere Disziplinen mit deren unterschiedlichen Arbeitsmitteln betreffen - werden zunehmend unverdaulich. Die Deutsche Gesellschaft für interdisziplinäre klinische Medizin (DGIKM) e.V. und deren Publikationsorgan klinikarzt fokussieren sich auf diese interdisziplinären Bereiche und bereiten diese auf, um sie für die Fort- und Weiterbildung verfügbar zu machen.

Sprechen wir von Interdisziplinarität, geht es nicht nur darum, mehrere Disziplinen mit ihren Arbeitsmethoden und Sachinhalten aneinander zu reihen. Vielmehr handelt es sich gerade bei den neuen Problemfeldern um Bereiche, die ganz neu zwischen den Disziplinen - was der Begriff Interdisziplinarität eigentlich bedeutet - angesiedelt sind und die Erarbeitung und Verfügbarkeit neuer Untersuchungsmethoden und damit neuer Verständnisprozesse erfordern.

Welche Auswirkungen werden diese neuen Entwicklungen auf die berufliche Fort- und Weiterbildung haben? Hierzu ein praktisches Beispiel: Wird ein in den Ingenieurwissenschaften diplomierter Wissenschaftler in der medizintechnischen Arbeitswelt eingesetzt, ist dies bislang institutionell nicht abgedeckt. Ohne spezifische, professionelle, harte Berufsfeldkenntnisse wird er jedoch nicht innovativ sein können, da seine Aufgabe beispielsweise ein Verständnis der Wechselwirkungen technischer Systeme mit Organen erfordert - wie dies zum Beispiel bei einem Defibrillator zur Wiederbelebung eines stillstehenden Herzens der Fall ist. Das Curriculum einer ingenieurswissenschaftlichen Ausbildung deckt diese Bereiche verständlicherweise nicht ab. Völlig realitätsfern wäre es jedoch, anzunehmen, dass solche Berufsfelder nur durch Personen getragen werden könnten, die sich mehreren Studien und Disziplinen gewidmet haben.

Es ist also nötig, im Rahmen der Weiterbildung in diesen Bereichen, das für die Berufswelt erforderliche Zusatzwissen in kurzer Zeit zu vermitteln. Dies erfordert moderne Trainingsprogramme, da sich die bisher klassische Vermittlung von Wissen nicht dazu eignet, in einer kurzen Zeit hochprofessionelle Qualität zu erzielen. Moderne Fort- und Weiterbildungssysteme bedürfen daher einer sorgfältigen, zeitökonomischen und zielgruppenorientierten Bearbeitung, die unter Vermeidung jeder Redundanz des Inhalts moderne Erkenntnisse des Lehr- und Lernverhaltens berücksichtigen.

Es ist aber nicht nur die Brisanz der nicht durch die studierte Disziplin zugänglichen Arbeitsfelder, sondern die auch innerhalb einer Disziplin selbst bestehenden Wissenslücken, die Beachtung finden müssen. Gerade in einer so komplexen Arbeitswelt wie der Medizin müssen - auch in Bezug auf die modernen Finanzierungssysteme wie das DRG-System - Arbeitsweisen weitergebildet werden, die über die konventionelle, disziplinorientierte Bearbeitung medizinischer Probleme weit hinausreichen.

Wir hoffen, in der DGIKM und über die Publikationsleistungen der Zeitschrift klinikarzt solche multiprofessionellen, interdisziplinären Erkenntnisse bereitstellen zu können, um den aktuellen Wissensstand im Bereich der Medizin effizient und interessant zu vermitteln.

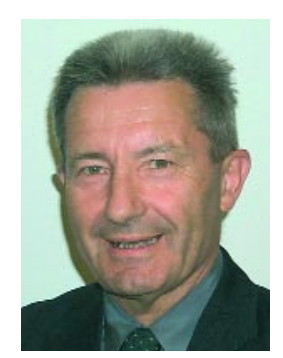

Prof. Dr. Dr. Dr. h.c. A. Grünert, Ulm

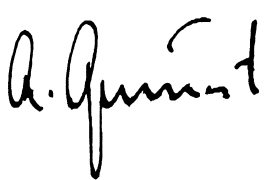

